\title{
Recent results from the second CDMSlite run and overview of SuperCDMS SNOLAB project
}

\author{
Tsuguo Aramaki ${ }^{*}$ \\ SLAC National Accelerator Laboratory/Kavli Institute for Particle Astrophysics and Cosmology, \\ Menlo Park, CA 94025, USA \\ E-mail: tsuguodslac.stanford.edu
}

The CDMS (Cryogenic Dark Matter Search) low ionization threshold experiment (CDMSlite) in the Soudan mine uses Ge detectors operated at a relatively high bias voltage, where the phonon signals are enhanced due to the Neganov-Luke effect. This allows us to lower the energy threshold for the low-mass dark matter search. The recent result of $70 \mathrm{~kg}$-days exposure in the second CDMSlite run excludes new parameter space in the dark matter mass range of $1.6-5.5 \mathrm{GeV}$. The SuperCDMS collaboration is now moving forward with the design and construction of the SuperCDMS SNOLAB project. Overview of the project and our progress to date are presented here.

The 11th International Workshop Dark Side of the Universe 2015

14-18 December 2015

Kyoto, Japan

\footnotetext{
*Speaker.

${ }^{\dagger}$ On behalf of the SuperCDMS Collaboration.
} 


\section{Overview}

The CDMS (Cryogenic Dark Matter Search) project has been leading the direct dark matter (DM) search for about the past 15 years. CDMS has uniquely explored the DM parameter space by measuring the nuclear recoil energy with both phonon (heat) and ionization (charge) signals. The phonon measurement, in particular, allows us to have a very low energy threshold since almost all the deposited energy in the crystal is converted into phonons.

The first CDMS project, CDMS I was operated at the Stanford Underground Facility [1], where cosmic-rays and their secondary particles including neutrinos were a major background for the direct DM search. The second generation of CDMS, CDMS II, was operated at the Soudan mine in Minnesota [2]. The third generation of CDMS, SuperCDMS Soudan, also operated in the Soudan mine with upgraded detectors and electronics [3, 4]. The SuperCDMS Soudan operation was completed in December 2015 with about $2500 \mathrm{~kg}$-days of exposure. The CDMS collaboration is now moving forward with the SuperCDMS SNOLAB project, planned to operate at SNOLAB near Sudbury in Ontario, Canada. The muon flux in SNOLAB is about three orders of magnitude smaller compared with the one at the Soudan site. We will use larger crystals and the exposure will be $140 \mathrm{~kg}$-years to further deeply investigate DM parameter space.

\section{SuperCDMS Soudan}
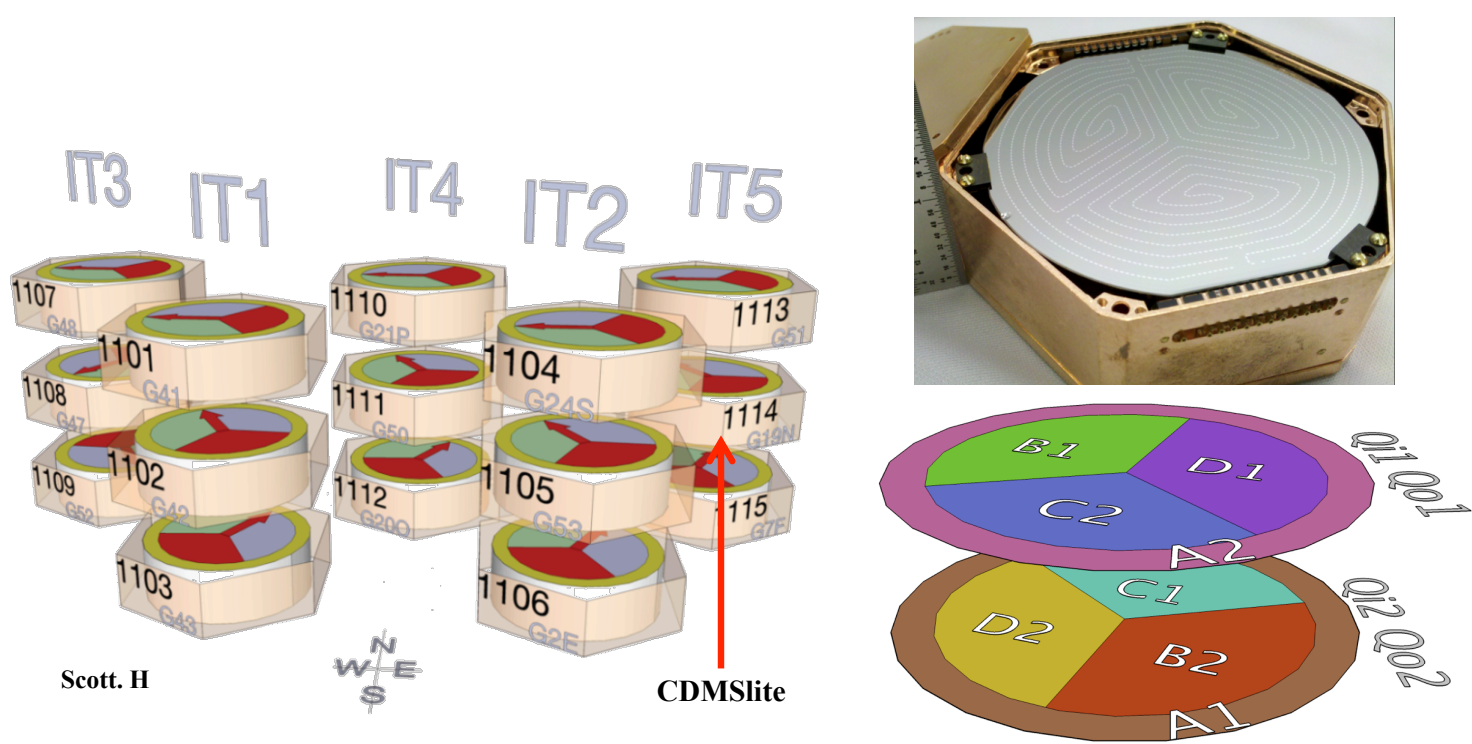

Figure 1: SuperCDMS Soudan detector and tower. There are five towers and each tower has three detectors.

In SuperCDMS Soudan, five towers were deployed. Each tower has three detectors and there are 15 detectors in total (see Fig. 1). The detectors were made of $7.5 \mathrm{~cm}$ diameter, $2.54 \mathrm{~cm}$ thick Ge crystals. Both phonon and ionization signals are used to measure the nuclear/electron recoil energy. Unlike CDMS II, phonon and ionization signals are read from both sides in SuperCDMS Soudan, which provides z-fiducialization inside the crystals. There are two ionization channels per side, an inner region and outer region, while there are four phonon channels per side, one 
in the outer ring and three in the inner region. In order to have better position reconstruction, sensor boundaries on the bottom side were rotated by 60 degree relative to the top side. One of the detectors was operated with higher voltage (called HV detector) for the CDMS low ionization threshold experiment (CDMSlite).

\subsection{Detection Concept}

As discussed above, both phonon and ionization signals are used to measure the recoil energy. When the DM particle scatters off the target nuclei, phonons and electron-hole pairs are created. Since the electric field is applied between the top and bottom electrodes, the electrons and holes are separated and drifted to each side. Here, the drifting electrons and holes create additional phonons, called Luke phonons due to the Neganov-Luke effect [5]. The ionization signals are read out with the charge sensitive amplifiers, while phonons are collected in the superconductive Al fins. In the Al fin, phonons break Cooper pairs and create quasi-particles (QPs). QPs, then, diffuse into W transition edge sensors (TESs) and transfer the energy. The W TESs are operated at the transition between superconductive and normal states. Therefore, as the energy is transferred into TESs, they are warmed up and the resistance sharply increases, which changes the current in the input coil. Since the input coil is coupled to SQUID arrays and the Feedback (FB) coil, the SQUID amplifier reads the phonon signal based on the magnetic flux induced by the current change in the input coil. This process is illustrated in Fig. 2.

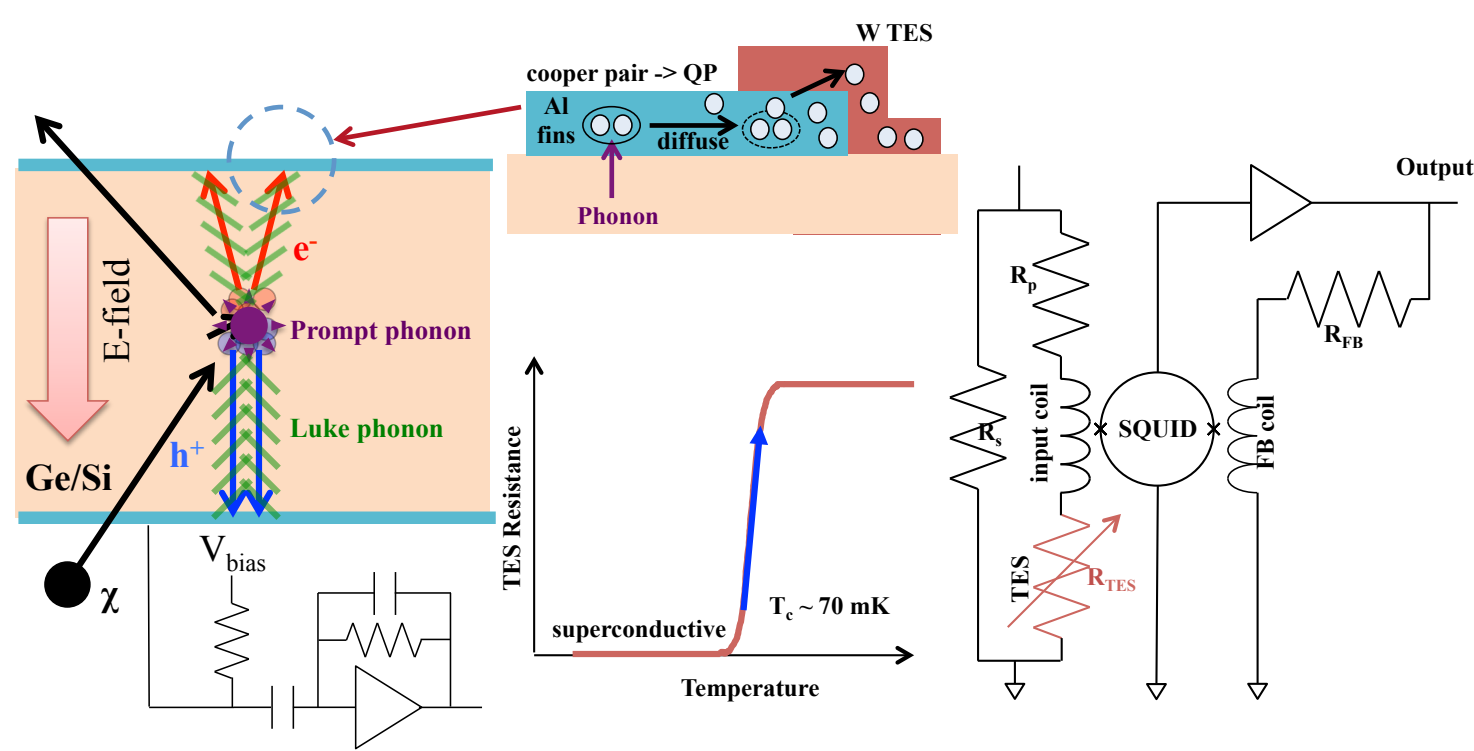

Figure 2: CDMS detection concept. The recoil energy is measured with ionization and phonon signals. The ionization signals are read by the charge sensitive amplifiers, while the phonon signal is measured with the TESs and the SQUID amplifier.

\subsection{Surface event rejection with ionization signals}

We have developed an interleaved Z-sensitive Ionization and Phonon (iZIP) detector, which can provide an excellent surface event rejection capability. This is critical to reduce background events since most of the electron recoil events such as low energy electrons and X-rays are surface 
events, while most of the nuclear recoil events from DM particles are bulk events. In the iZIP detector, the phonon sensors are grounded, while the electrodes for the ionization signals are biased with $+2 \mathrm{~V}$ at the electrode on the top side and $-2 \mathrm{~V}$ on the bottom side. Therefore, this configuration creates electric fields between top and bottom sides, as well as between the phonon sensor and the electrode near the surface. As a result, the electrons are drifted to the top surface for bulk events, while both electrons and holes are collected on the bottom side for the bottom surface events (see left and middle figures in Fig. 3). This will allow us to distinguish bulk events from surface events, based on the ionization signals at side- 1 and side-2 (see the right figure in Fig. 3). Blue dots are bulk events, which shows symmetrical signals between side- 1 and side-2, while black dots are surface events, which shows asymmetrical signals between side- 1 and side- 2 .
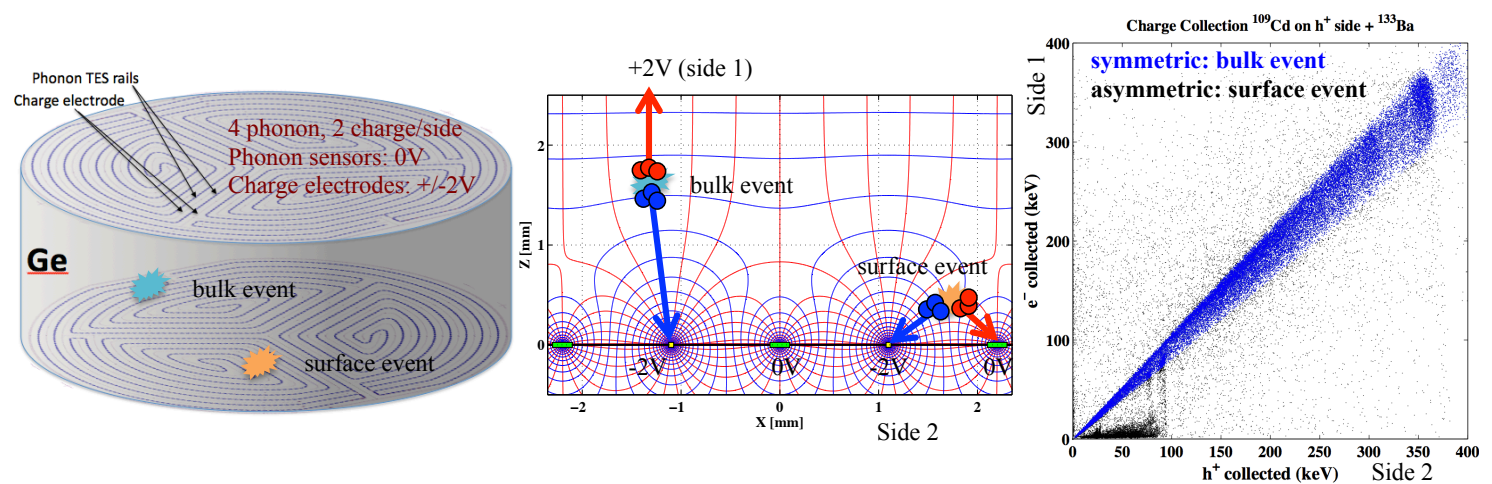

Figure 3: Surface event rejection with ionization signals. Figures are taken from [6].

\subsection{Surface event rejection with phonon signals}

In addition to the ionization signals, the phonon pulse shape also provides a surface rejection capability [7]. Phonons usually propagate isotropically and bounce many times before collected in the Al fin. For surface events, however, some phonons will be collected without multiple bounces and a sharp peak can be seen at the beginning of the pulse shape, unlike bulk events. Therefore, based on the pulse shape and the signals at side- 1 and side-2, we can distinguish surface events from bulk events (see Fig. 4).

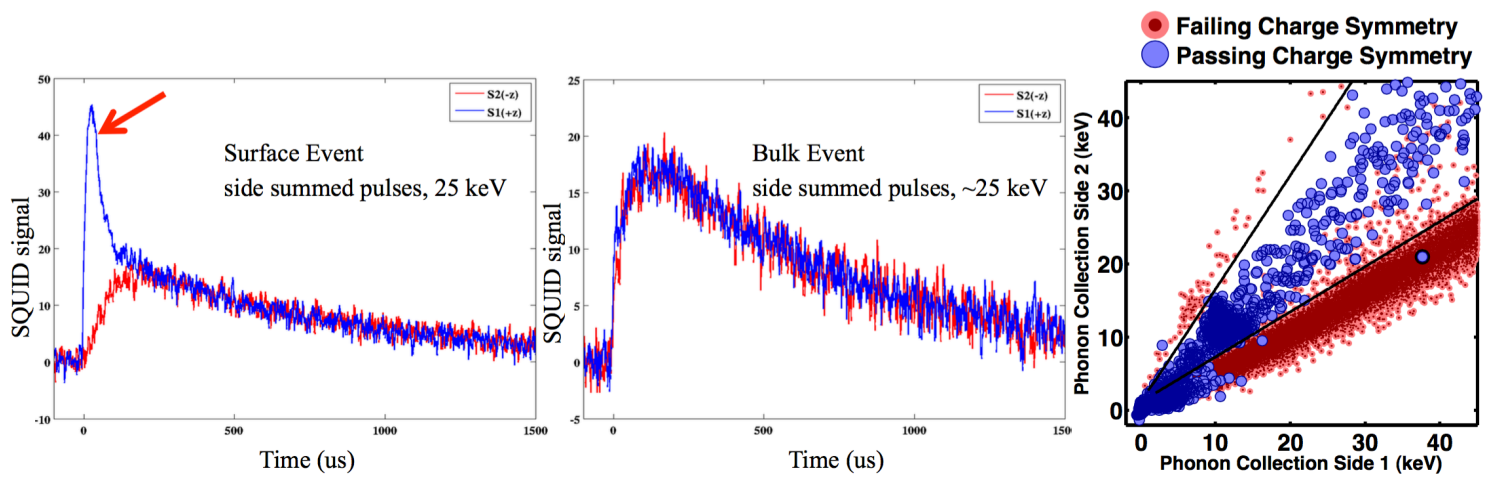

Figure 4: The phonon pulse shape and side-1 and side- 2 signals for surface and bulk events. Unlike bulk event, a sharp peak can be seen in the pulse shape and signals between side- 1 and side- 2 are asymmetrical for surface events. Figures are taken from [6, 7]. 


\subsection{Ionization yield and nuclear recoil event selection}

The iZIP detector also provides an excellent capability to distinguish nuclear recoil events from electron recoil events. In a nuclear recoil event, the recoiled target nuclei quickly stops and creates fewer e-h pairs, compared with an electron recoil event (see the left figure in Fig. 5). As a consequence, the ratio of the ionization energy to the recoil energy for the nuclear recoil events becomes smaller. This ratio is called ionization yield and normalized as $\sim 1$ for electron recoil events and $\sim 0.3$ for nuclear recoil events, based on the Lindhard theory [8]. Note that the recoil energy can be reliably calculated from the phonon signals. The right figure in Fig. 5 shows the calibration results, where the blue dots are the ionization yield of bulk electron recoil events, while the green lines show the $2 \sigma$ band for the nuclear recoil events obtained by the ${ }^{252} \mathrm{Cf}$ calibration source [6]. The red dots are identified as surface events, based on the ionization signals at side-1 and side-2, as discussed above. The hyperbolic black line is the ionization threshold (1.6 keVee) and the vertical black line is the recoil energy threshold $(8 \mathrm{keVr})$.
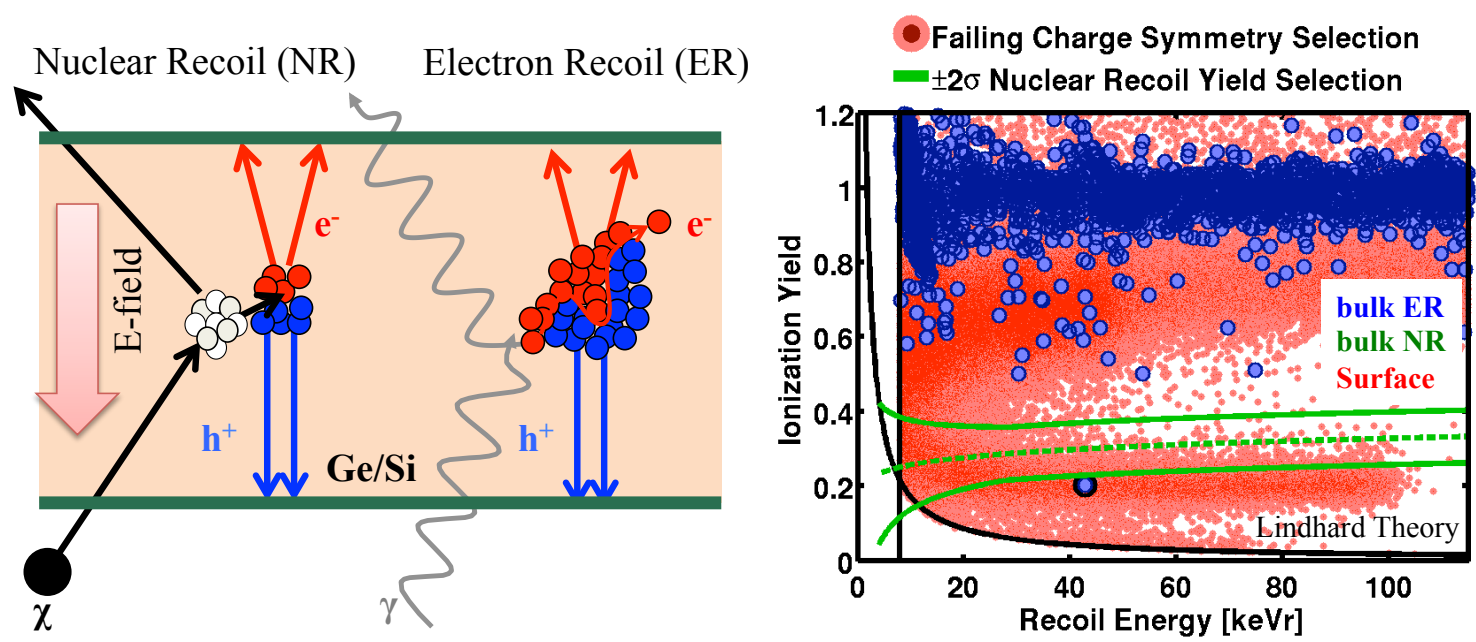

Figure 5: Ionization yield for nuclear recoil and electron recoil events. The right figure is taken from [6].

\subsection{CDMSlite second run}

One of the iZIP detectors was operated in HV mode (CDMSlite) to measure the low energy signal from the low-mass DM interaction. The 70V bias voltage was applied to side-2, while side-1 was grounded. The phonon signal was read only from side- 1 . The enhanced phonon signal due to the Neganov-Luke effect can be estimated as below;

$$
E_{p t}=E_{r}\left(1+Y \frac{q V}{\varepsilon}\right)
$$

Here, $E_{p t}$ is the total phonon energy, $E_{r}$ is the recoil energy, $Y$ is the ionization yield, $q$ is the elementary charge, $V$ is the applied voltage and $\varepsilon$ is the required energy to create a electron-hole pair. The second term on the right hand side is the Luke phonon signal, which increases as the applied electric field becomes larger. With the 70V bias voltage, the energy threshold becomes 10 times smaller and the electron recoil background events can be reduced by a factor of 5 . The left figure in Fig. 6 shows the energy spectrum, where we were able to identify the ${ }^{71}$ Ge activation 
lines (10.4 keV for K-shell, $1.30 \mathrm{keV}$ for L-shell and $0.16 \mathrm{keV}$ for M-shell). We also successfully demonstrated that the phonon signal was not degraded when we increased the bias voltage up to 90 V. Note that as we only focused on the phonon signals in CDMSlite, we were not able to distinguish nuclear recoil events from electron recoil events with the ionization yield.
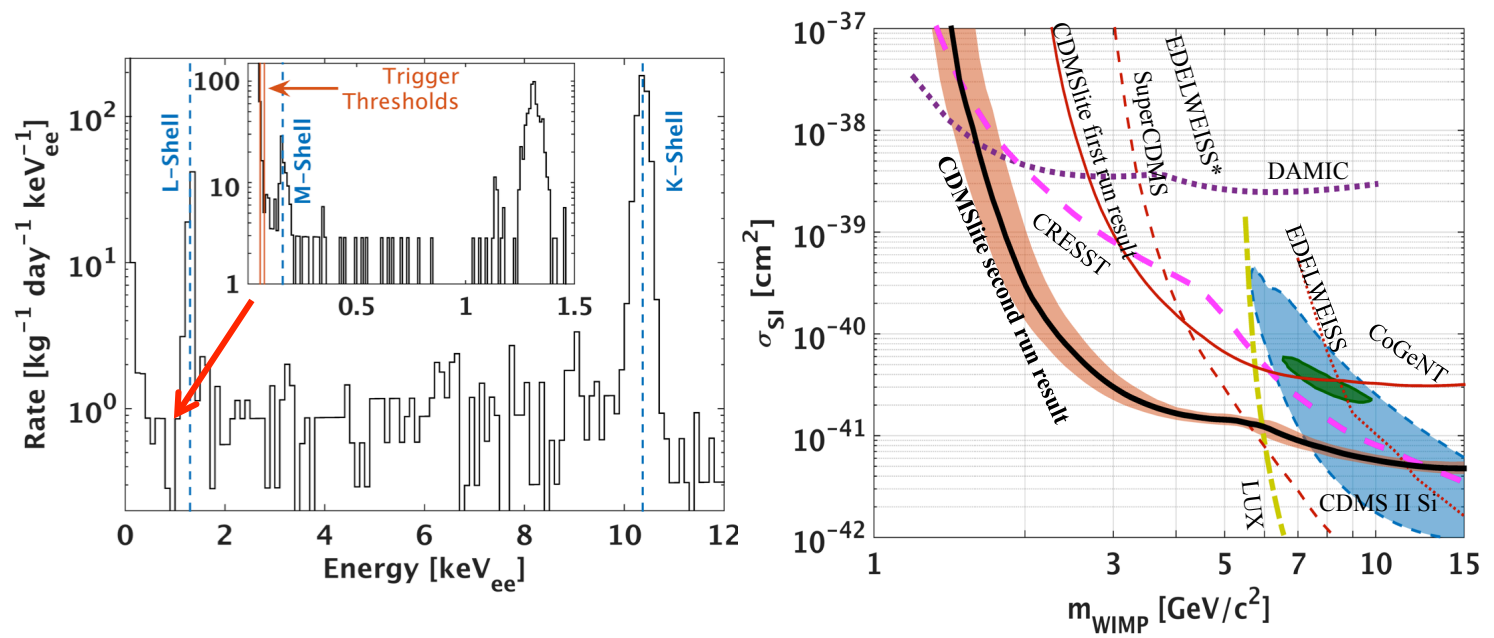

Figure 6: Left: Energy spectrum after selection cuts applied. The ${ }^{71} \mathrm{Ge}$ activation lines, $10.4 \mathrm{keV}$ (K-shell), $1.30 \mathrm{keV}$ (L-shell) and $0.16 \mathrm{keV}$ (M-shell), as well as the trigger threshold are shown in the figure. Right: The upper limits for the spin-independent DM-nucleon cross-section obtained from the CDMSlite second run (red: $90 \%$ C.L., black: 95\% C.L.), as well as the ones from the first CDMSlite run, SuperCDMS, EDELWEISS-II, LUX, CRESST, and DAMIC (90\% C.L.). Closed regions are possible DM signals obtained from CDMS II Si and CoGeNT (90\% C.L.). Figures are taken from [9].

Since the accelerometer sensor was installed after the first run, we were able to reduce the low frequency noise in the second run. With the better energy calibration and new radial fiducial cut, we were able to achieve the electron recoil energy threshold as low as $\sim 60 \mathrm{eV}$ (see $[9,10,11]$ for details). As a result, we obtained an order of magnitude better exposure and sensitivity from the second run, which excludes the new parameter space of the DM-nucleon spin-independent cross section, in the DM mass range of $1.6-5.5 \mathrm{GeV}$ (see the right figure in Fig. 6). The upper limits for the spin-independent DM-nucleon cross-section obtained from the first CDMSlite run, SuperCDMS, EDELWEISS-II, LUX, CRESST, and DAMIC, as well as the closed regions for the possible DM signals from CDMS II Si and CoGeNT are shown in the figure [9].

\section{SuperCDMS SNOLAB}

The SuperCDMS SNOLAB project was selected as a DOE/NSF second-generation direct DM search program and we are currently engaging on the design and construction of the SuperCDMS SNOLAB detector and tower. Initially 5 towers will be deployed, which consist of 3 Ge iZIP towers, $1 \mathrm{Si}$ iZIP tower and one HV tower. Each tower will have six detectors that are made of $10 \mathrm{~cm}$ diameter $3.3 \mathrm{~cm}$ thick Ge/Si crystals. The left figure in Fig. 7 shows the prototype detector and tower for SuperCDMS SNOLAB. We will reduce the background events by minimizing Radon exposure time and cosmogenic activation, and using cleaner copper parts. Furthermore, by lowering the detector $T_{c}$ and using low-power low-noise electronics, such as new SQUID arrays with a 
gradiometric input for phonon readout and HEMTs (High Electron Mobility Transistors) for ionization readout, energy resolution for phonon and ionization signals will be significantly improved [12].

\subsection{Detector Design}

In SuperCDMS SNOLAB, the iZIP detector will have six phonon and two ionization channels per side. For the HV detectors, the Luke phonons can provide z-fiducialization, based on the signals between side- 1 and side- 2 . We are currently optimizing the mask design for the HV detector.
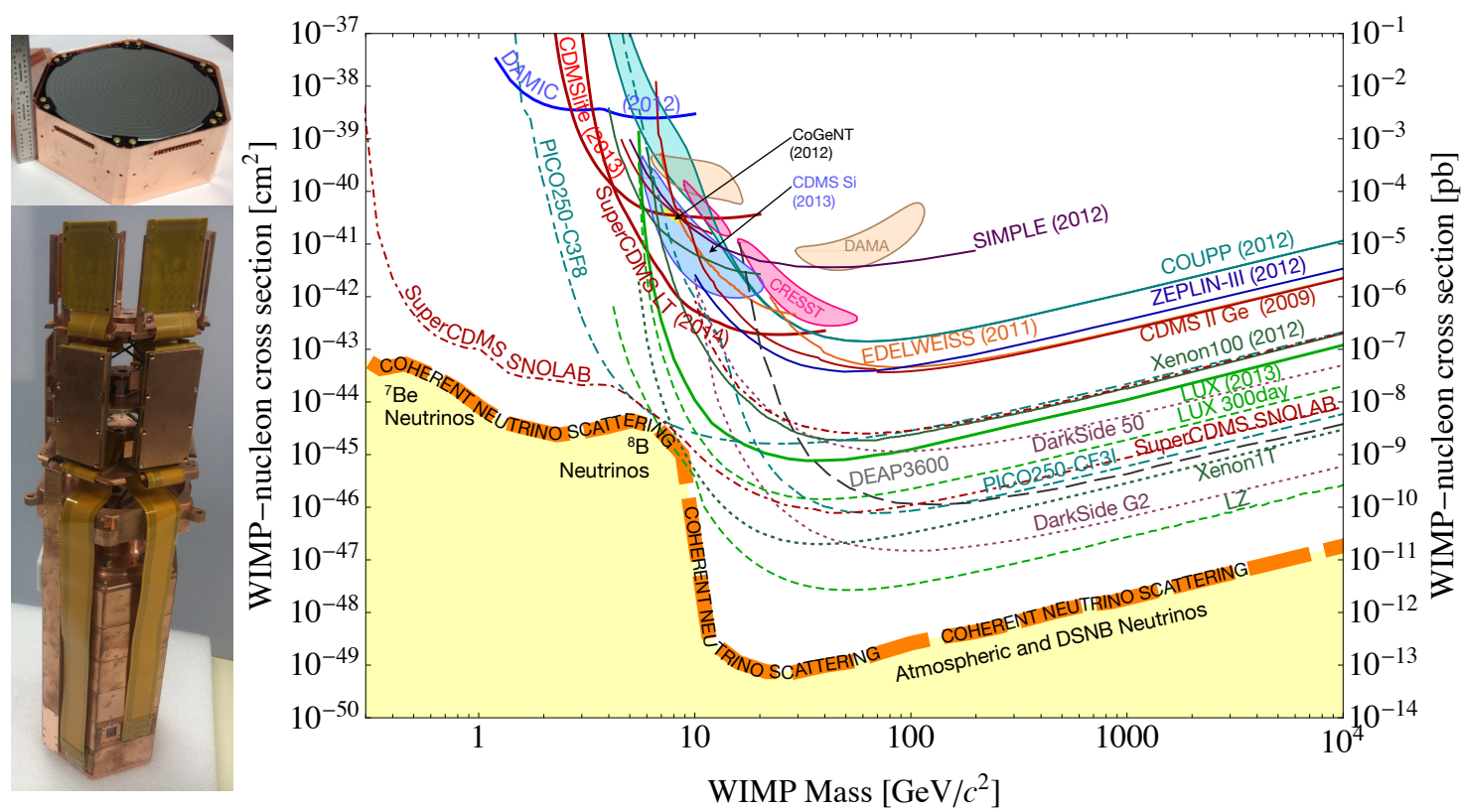

Figure 7: Left: SuperCDMS SNOLAB detector and tower. Each tower has six detectors that are made of 10 $\mathrm{cm}$ diameter $3.3 \mathrm{~cm}$ thick Ge/Si crystals. Right: SuperCDMS SNOLAB sensitivity. The right figure is taken from [12].

\subsection{Sensitivity}

The right figure in Fig. 7 shows the sensitivity of the SuperCDMS SNOLAB project (140 kg-years exposure) in the DM parameter space, DM mass and DM-nucleon spin-independent interaction cross section. SuperCDMS SNOLAB can uniquely and deeply probe the parameter space especially for low-mass DM regions (below $5 \mathrm{GeV}$ ), and it can complement other direct DM search experiments, such as LZ and XENON for DM mass above $10 \mathrm{GeV}$ [12]. Furthermore, the mixture of the Ge and Si targets in SuperCDMS will allow us to study non-standard DM-nucleon interaction, such as the isospin-violation model in the effective field theory [13]. We are also expecting to detect about $15^{8} \mathrm{~B}$ solar neutrinos [12].

\section{Conclusion}

The CDMS project has been leading the direct DM search for about the past 15 years, while deeply probing the DM parameter space. One of the iZIP detectors in SuperCDMS Soudan was 
operated with high voltage, which allowed us to lower the energy threshold and exclude new parameter space in the DM mass range of $1.6-5.5 \mathrm{GeV}$.

We are moving forward with the next generation of the CDMS project, SuperCDMS SNO$\mathrm{LAB}$, where the larger exposure, reduced background and upgraded detectors and electronics will allow us to further deeply investigate the DM parameter space. In particular, we will uniquely probe the parameter space for low-mass DM regions (below $5 \mathrm{GeV}$ ), while complementarily covering higher DM mass regions (above $10 \mathrm{GeV}$ ) with other direct DM search experiments.

\section{Acknowledgements}

The SuperCDMS collaboration gratefully acknowledges the contributions from the staff of the Soudan Underground Laboratory and the Minnesota Department of Natural Resources. Part of the research for CDMSlite was conducted under the Ultra Sensitive Nuclear Measurements Initiative at Pacific Northwest National Laboratory, operated by Battelle for the U.S. Department of Energy. The iZIP detectors were fabricated in the Stanford Nanofabrication Facility, a member of the National Nanofabrication Infrastructure Network. This work is supported by the National Science Foundation, the United States Department of Energy, Fermilab, NSERC Canada, and MultiDark (Spanish MINECO). Fermilab is operated by the Fermi Research Alliance, LLC under Contract No. De-AC02-07CH11359. SLAC is operated under Contract No. DEAC02-76SF00515 with the United States Department of Energy.

\section{References}

[1] D.S. Akerib et al., Phys. Rev. D 68.8082002 (2003).

[2] R. Agnese et al., Phys. Rev. Lett. 111, 251301 (2013).

[3] P.L. Brink et al., astro-ph/0503583 (2005).

[4] D.S. Akerib et al., Nucl. Instr. Meth. Phys. Res. A 559.2, 411-413 (2006).

[5] P. N. Luke, Appl. Phys. Lett. 65, 2884 (1994).

[6] R. Agnese et al., Appl. Phys. Lett. 103, 164105 (2013).

[7] M. Pyle, Ph.D. thesis, Stanford University (2012).

[8] J. Lindhard et al., Mat. Fys. Medd. Dan. Vid. Selsk. 33, No.10 (1963).

[9] Agnese, R., et al., Phys. Rev. Lett. 116.7, 071301 (2016).

[10] R. Agnese et al., Phys. Rev. Lett. 112, 041302 (2013).

[11] R. Agnese et al., Phys. Rev. Lett. 112, 241302 (2014).

[12] A.E. Robinson presented at APS 2016, S. Galowala, presented at UCLA Dark Matter 2016.

[13] K. Schneck et al., Phys. Rev. D 91.9092004 (2015). 